\title{
Selenium supplementation for patients with Graves' hyperthyroidism (the GRASS trial): study protocol for a randomized controlled trial
}

Torquil Watt ${ }^{*}$, Per Cramon ${ }^{1}$, Jakob Bue Bjorner ${ }^{2,3}$, Steen Joop Bonnema ${ }^{4}$, Ulla Feldt-Rasmussen ${ }^{1}$, Christian Gluud ${ }^{5}$, Jeppe Gram ${ }^{6}$, Jane Lindschou Hansen ${ }^{5}$, Laszlo Hegedüs ${ }^{4}$, Nils Knudsen", Pernille Bach-Mortensen ${ }^{8}$, Runa Nolsøe ${ }^{9}$, Birte Nygaard ${ }^{10}$, Flemming Pociot ${ }^{11}$, Maria Skoog ${ }^{5}$, Per Winkel ${ }^{5}$ and Åse Krogh Rasmussen ${ }^{1}$

\begin{abstract}
Background: Graves' hyperthyroidism is an autoimmune disease causing hyperfunction of the thyroid gland. The concentration of selenium is high in the thyroid gland and two important groups of enzymes within the thyroid are selenoproteins, that is, they depend on selenium. Selenium may have beneficial effects on autoimmune hypothyroidism and on Graves' orbitopathy, but the effects of selenium on Graves' hyperthyroidism is unknown. We hypothesize that adjuvant selenium may be beneficial in the treatment of Graves' hyperthyroidism. The objective is to investigate if selenium supplementation plus standard treatment with anti-thyroid drugs versus standard treatment with anti-thyroid drugs will lead to a decrease in anti-thyroid drug treatment failure (that is, failure to remain euthyroid, without further treatment, one year after cessation of anti-thyroid drug treatment), faster and longer lasting remission (that is, anti-thyroid drug treatment success), and improved quality of life in patients with Graves' hyperthyroidism.
\end{abstract}

Methods and design: The trial is an investigator-initiated, randomised, blinded, multicentre clinical trial. Inclusion criteria are: age 18 years or older; diagnosis of active Graves' hyperthyroidism within the last two months; and informed consent. Exclusion criteria are major co-morbidity; previous radioactive iodine treatment; ongoing anti-thyroid drug treatment for more than two months; treatment with immunomodulatory drugs; known allergy towards the components in the selenium and placebo pills; pregnancy or breast-feeding; and intake of selenium supplementation above $70 \mu \mathrm{g}$ per day. We plan to include 492 participants, randomised (1:1) to two tablets of $100 \mu \mathrm{g}$ selenium once daily for the 24 to 30 months intervention period versus two identical placebo tablets once daily. The primary outcome is the proportion of participants with anti-thyroid drug treatment failure (see above) at the end of the intervention period (24 to 30 months). Secondary outcomes are: thyroid-specific quality of life during the first year after randomisation; level of thyroid stimulating hormone-receptor antibodies at 18 months after randomisation and at the end of the intervention period (24 to 30 months); hyperthyroid symptoms during the first year after randomisation; eye symptoms during the first year after randomisation, and at the end of the intervention period (24 to 30 months); adverse reactions during the intervention period; and serious adverse events during the intervention period.

(Continued on next page)

\footnotetext{
* Correspondence: T.Watt@rh.dk

'Department of Medical Endocrinology, Copenhagen University Hospital

Rigshospitalet, Blegdamsvej 9, DK-2100 Copenhagen, Denmark

Full list of author information is available at the end of the article
} 
(Continued from previous page)

Discussion: It was of great importance to the initiators of this trial, that the results would be directly applicable to daily clinical practice. Therefore, it was designed as a pragmatic trial: the patients follow their usual treatment at their usual hospitals. In order to still collect high quality data on the clinical course and quality of life, an elaborate trial management system was designed to keep track of patient input, need for trial personnel input and action, and to collect data from medical chart systems. Meticulous follow-up on missing responses to the QoL measurements has been incorporated into the system, to minimise missing quality of life data. Monitoring of adverse reactions and events is achieved by thorough instruction of the participants, surveillance of patient-reported outcomes, and integration with national databases regarding hospitalizations. A very long intervention period was necessary, since patients are not considered in remission until one year after stopping anti-thyroid drugs. Usually, patients are treated for 12 to 18 months with anti-thyroid drugs, yielding a total intervention period of 24 to 30 months.

Trial registration: ClinicalTrials.gov: NCT01611896.

Keywords: Graves' disease, Selenium supplementation, Pragmatic trial, Quality of life, ThyPRO

\section{Background}

Graves' hyperthyroidism is an autoimmune disease causing hyperfunction of the thyroid gland by a mechanism, where auto-antibodies bind to and stimulate the thyroid stimulating hormone (TSH) receptor. It affects individuals of all ages and is five to ten times more common in women than in men, with an overall annual incidence in Denmark of about 1,700 patients in 5.5 million people [1]. Treatment of Graves' hyperthyroidism comprise: 1) anti-thyroid drugs (ATD) (for example, methimazole or propylthiouracil); 2) thyroidectomy, that is, surgical removal of the thyroid gland, and 3) radioactive iodine treatment $\left(\mathrm{I}^{131}\right)$, which permanently reduces thyroid function. In Europe, the primary treatment is usually ATD, which leads to resolution of hyperthyroidism (that is, euthyroidism) in $85 \%$ to $90 \%$ of patients within 6 weeks [2]. This treatment is usually continued for a period of 12 to 18 months and then tapered off (ATD treatment withdrawal). However, $30 \%$ to $60 \%$ of patients will experience relapse of hyperthyroidism during the following years [2]. Patients with Graves' hyperthyroidism suffer from a wide range of symptoms and have major impairments in most areas of quality of life (QoL) [3].

Selenium is an essential trace element important for human health. The main dietary sources of selenium in Denmark are meat, poultry, dairy products, bread, cereals and fish $[4,5]$. The recommended daily intake of selenium is $40 \mu \mathrm{g}$ for women and $50 \mu \mathrm{g}$ for men. The estimated actual daily intake in Denmark is considered sufficient, but it cannot be ruled out that about $10 \%$ of the Danish population could benefit from selenium supplementation [4]. The upper tolerable level of selenium intake is set to $400 \mu \mathrm{g}$ per day in the USA [6] and $300 \mu \mathrm{g}$ per day in the EU [7].

The thyroid gland has the highest selenium concentration per unit weight among all tissues. Selenium is incorporated into key enzymes involved in several metabolic pathways. The main selenoprotein families are the glutathione peroxidases, the thioredoxin reductases and the iodothyronine deiodinases [5]. It is hypothesized that the glutathione peroxidases and the thioredoxin reductases participate in a complex defence system maintaining normal thyroid function by protecting the gland from both hydrogen peroxide, which is produced by the thyrocytes, and reactive oxygen intermediates [8,9]. It has thus been hypothesized, that selenium may have a beneficial role in autoimmune thyroid diseases, by blunting the autoimmune process.

We have not identified any published randomised trials of selenium supplementation in patients with Graves' hyperthyroidism. On ClinicalTrials.gov, one ongoing randomised trial is registered on selenium supplementation in Graves' thyrotoxicosis (NCT01247077). This trial assesses neuropsychological well-being (not otherwise specified), after nine months intake of $200 \mu \mathrm{g}$ selenium, and includes 44 participants. In a multicentre trial among 159 patients with mild Graves' orbitopathy, without hyperthyroidism, $200 \mu \mathrm{g}$ selenium selenite improved diseasespecific QoL $(P<0.001)$ and reduced eye disease severity $(P=0.01)[10]$. Another trial evaluated the effect of adding a mixture of antioxidants, including $60 \mu \mathrm{g}$ of selenium (not otherwise specified) to standard ATD treatment in 29 patients with Graves' disease. During the 60-day follow up period euthyroidism was reached more rapidly in patients receiving antioxidants [11-13]. In contrast, several randomised trials have evaluated the effect of selenium on the other major autoimmune thyroid disease: autoimmune hypothyroidism. In six [14-19] of seven [14-20] placebo-controlled clinical trials, selenium treatment reduced thyroid peroxidase antibody (TPOAb) levels, indicating a beneficial effect on the autoimmune activity.

We hypothesize that the addition of selenium supplementation to the standard treatment with ATD in patients with active Graves' hyperthyroidism will lead to a decrease in ATD treatment failure (that is, fewer patients with relapse), faster remission, and improved quality of life. 


\section{Methods and design Objectives}

The primary objective is to investigate the effect of selenium supplementation on the proportion of participants with ATD treatment failure, that is, failure to remain euthyroid without further treatment one year after cessation of ATD treatment.

The secondary objectives are to investigate the effect on thyroid-specific QoL, level of TSH-receptor antibody (TRAb), hyperthyroid symptoms, eye symptoms, adverse reactions, and serious adverse events. Further, we wish to explore the effect of selenium on ATD treatment duration, incidence of Graves' orbitopathy, and hypothyroid symptoms.

\section{Design}

The GRASS (GRAves' disease Selenium Supplementation trial) trial is an investigator-initiated, randomised, blinded, multicentre clinical trial of selenium supplementation versus placebo in patients with Graves' hyperthyroidism. The trial has a parallel-arm design with 1:1 allocation to the experimental intervention group and the control intervention group, and involving seven clinical trial sites in Denmark (see Figure 1). The trial also includes a questionnaire and register-based follow up period up to ten years after completion of the intervention period.

\section{Trial participants}

All patients with current hyperthyroidism, who are referred to or being followed at the participating clinical trial sites, are considered for participation. Patients are eligible for the GRASS trial, if they comply with the following inclusion and exclusion criteria.

\section{Inclusion criteria}

The inclusion criteria are: Graves' hyperthyroidism (firsttime diagnosis, defined as patients not yet receiving ATD treatment, or having received ATD treatment continuously for less than two months, or relapse of Graves' hyperthyroidism defined as patients previously having received and discontinued treatment with ATD); active Graves' hyperthyroidism (TSH $<0.1 \mathrm{mU} / \mathrm{L}$ and positive TRAb according to local laboratory results) measured within the last two months prior to the inclusion date; age 18 years or older; provision of written informed consent.

\section{Exclusion criteria}

The exclusion criteria are: major co-morbidity, rendering the participants unlikely to continuously receive the trial intervention; previous treatment with radioactive iodine; ongoing ATD treatment for more than two months; treatment with immunomodulatory drugs, such as cyclosporine A, methotrexate, cyclophosphamide; allergy to the components in the selenium and placebo pills; pregnancy or breast-feeding; intake of selenium supplementation above $70 \mu \mathrm{g}$ per day; inability to read and understand Danish; lack of provision of informed consent.

\section{Trial intervention \\ Selenium}

The compound used in this trial is organic selenium, in the form of selenium yeast, which mainly consists of selenomethionine. The specific product is Organisk selen, $100 \mu \mathrm{g}$ tablets and is produced by Jemo-Pharm A/S, Stege, Denmark (http://www.jemo-pharm.dk/frame.cfm/ $\mathrm{cms} / \mathrm{id}=977 / \mathrm{sprog}=2 / \mathrm{grp}=6 / \mathrm{menu}=1 /$ ). The daily dose is set at $200 \mu \mathrm{g}$ (two tablets taken in the morning). The trial dosage is based on the available clinical data, is not considered to cause adverse reactions, and is lower than the upper tolerable intake level of $300 \mu \mathrm{g}$ per day [6,7].

\section{Placebo}

Placebo tablets, identical in size, appearance, taste, smell, and solubility to the experimental intervention tablet are produced by Jemo-Pharm A/S. They have the same content as Organisk selen but are without selenium, as the selenium yeast has been exchanged with yeast grown in selenium-deplete media. The placebo regimen is identical to the selenium regimen.

\section{Randomisation}

Randomisation will be performed centrally. The allocation sequence is computer-generated with a varying block size kept unknown to the investigators. Randomisation is stratified by clinical trial site and disease status (incident or relapse), and the allocation ratio is $1: 1$.

\section{Informed consent procedure}

Potential participants are identified at referral or visits to the outpatient clinics and include all patients with current hyperthyroidism (that is, elevated thyroid hormone levels). Where Graves' hyperthyroidism is confirmed (that is, TRAb is positive), the potential participant is invited to an information visit by letter. The visit consists of obtaining history, blood sampling, information about trial contact, and randomisation.

\section{Duration}

The intervention period for each participant will be 24 to 30 months, as selenium will be given until 12 months after cessation of ATD treatment, which usually lasts 12 to 18 months (Figure 2). The total trial duration is expected to be about 4 years (inclusion period about 17 months and up to 30 months intervention period).

\section{Concomitant medication or treatment}

The trial participants receive conventional ATD treatment. Treatment of hyperthyroidism, will take place within the 

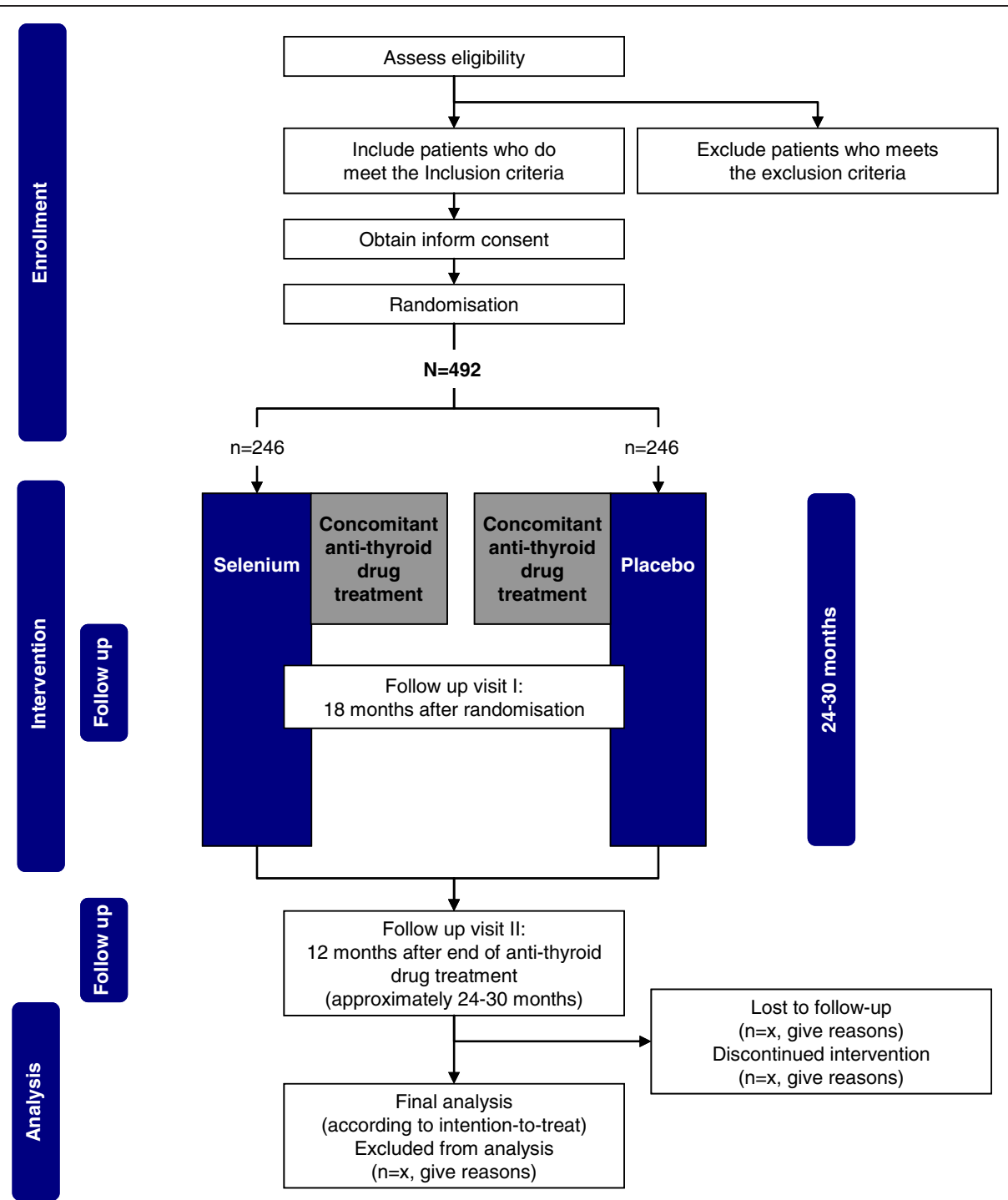

Figure 1 Trial flow chart.

participating clinical trial sites, according to the clinical standards set by the departments. ATD treatment withdrawal must be considered or attempted 18 months after randomisation at the latest.

Participants are advised not to take extra selenium supplementation during the trial. A dose of $<70 \mu \mathrm{g}$ per day is allowed (the content in multivitamin tablets is $55 \mu \mathrm{g}$ ). The participants' consumption of additional selenium supplements will be monitored during the trial through self-report forms.

\section{Monitoring for intervention compliance}

Participant compliance with the intervention will be monitored by self-reported tablet intake at 6 and 12 weeks, and 6,12 and 24 months (Table 1), and by tablet-counting at trial visits at 18 months and at the end of the intervention (24 to 30 months). An investigator will contact the patient if the trial data management system flags a participant as non-compliant (or overdosing), according to self-report of the number of unopened containers.

\section{Discontinuation}

A participant who no longer wishes to participate in the trial can withdraw his/her informed consent at any time without need of further explanation, and this will not have any consequences for the participant's further treatment. In order to conduct intention-to-treat analyses with as few missing data as possible, the investigator may ask the participants which aspects of the trial, they wish to withdraw from. These can include the following: 


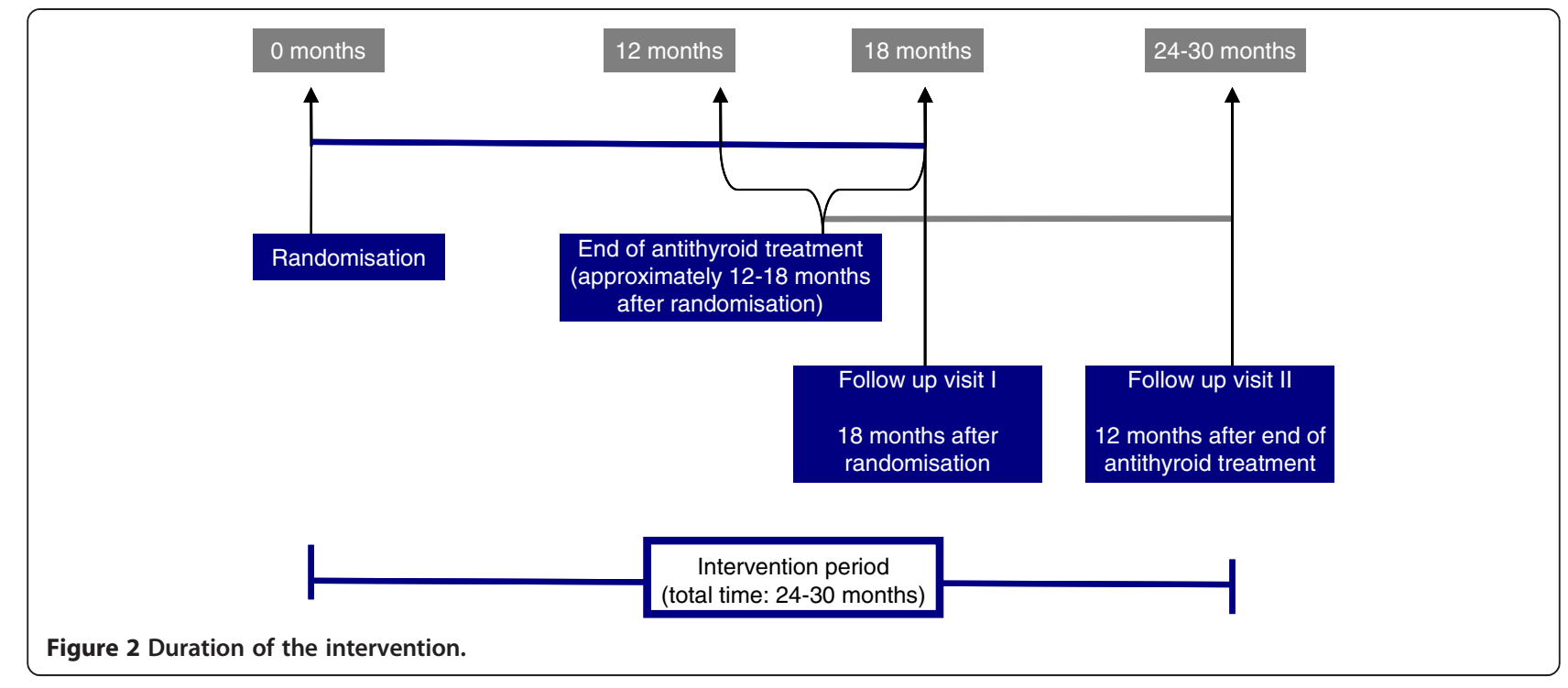

receipt of the trial intervention; participation in the remaining follow up assessments, and analysis of data already collected. The investigators will discontinue a participant's taking of the trial intervention at any time, if the participant: experiences intolerable adverse reactions; is diagnosed with any of the exclusion criteria during the intervention period; is referred for ablative therapy (radioactive iodine or thyroid surgery) during the intervention period. In all three cases, the investigator and/or the treating physician will, if possible, encourage the participant to continue with follow up assessment and to allow the use of collected data in the analyses.

\section{Blinding}

Blinding will be maintained for all parties in the trial, throughout all aspects of the trial. The trial interventions will be identical, and will be packed in identical packages by the Capital Region Pharmacy, and therefore, knowledge

Table 1 Trial schedule for assessments

\begin{tabular}{|c|c|c|c|c|c|c|c|c|}
\hline \multirow[b]{2}{*}{ Variable } & \multirow[b]{2}{*}{$\begin{array}{l}\text { Inclusion } \\
\text { (baseline) }\end{array}$} & \multicolumn{7}{|c|}{ Follow up } \\
\hline & & $\begin{array}{c}6 \\
\text { weeks }\end{array}$ & $\begin{array}{c}12 \\
\text { weeks }\end{array}$ & $\begin{array}{c}6 \\
\text { months }\end{array}$ & $\begin{array}{c}12 \\
\text { months }\end{array}$ & $\begin{array}{c}18 \\
\text { months }\end{array}$ & $\begin{array}{c}24 \\
\text { months }\end{array}$ & $\begin{array}{l}12( \pm 1) \text { months after ATD } \\
\text { treatment withdrawal }^{+}\end{array}$ \\
\hline Visit & $x$ & & & & & $x$ & & $x$ \\
\hline ATD treatment & & & & & & $x$ & $x$ & $x$ \\
\hline Thyroid function & $x^{1}$ & & & & & $x^{1}$ & & $x^{1}$ \\
\hline TSH-Receptor Antibodies & $x^{1}$ & & & & & $x_{s}$ & & $x_{s}$ \\
\hline Serum selenium & $x$ & & & & & $\left(x_{s}\right)$ & & $\left(x_{s}\right)$ \\
\hline Creatinine/iodine ratio in spot urine & $x$ & & & & & & & \\
\hline Storage samples (blood and urine) & $x$ & & & & & $x$ & & $x$ \\
\hline ThyPRO & $x_{r}$ & $x_{r}$ & $x_{r}$ & $x_{r}$ & $x_{r}$ & $x_{r}$ & $x_{r}$ & $x_{r}$ \\
\hline Tablet count & & $x_{r}$ & $x_{r}$ & $x_{r}$ & $x_{r}$ & $x_{r}$ & $x_{r}$ & $x_{r}$ \\
\hline Consumption of additional selenium & & & & $x_{r}$ & $x_{r}$ & $x_{r}$ & $x_{r}$ & \\
\hline Adverse events* & & $x_{r}$ & $x_{r}$ & $x_{r}$ & $x_{r}$ & $x_{r}$ & $x_{r}$ & $x_{r}$ \\
\hline Serious adverse events & & & & & & & & $x$ \\
\hline Referral to ablative therapy & & & & & & $x$ & & $x$ \\
\hline
\end{tabular}

All assessments must be made at the time points specified above. If the assessment is not possible at the specified time, the assessment shall still be conducted, and the time shall be noted in the electronic case report form (eCRF). Following this, deviations from the protocol can be assessed. ${ }^{1}$ As part of usual clinical practice $\left(f=\right.$ free (non-protein bound)), that is, all measured analyses are collected continuously from medical systems; $x_{s}$, analysed on stored plasma/serum samples after trial completion; $x_{r}$, self-reported data by participant; ${ }^{\dagger}$ participants for whom ATD treatment withdrawal has not been attempted, or has been unsuccessful, will be followed up at 36 months after randomisation ( \pm 1 month). ATD, anti-thyroid drugs; TSH, thyroid stimulating hormone; ThyPRO, thyroid patient-reported outcome. 
of allocated intervention group will be unknown to participants and investigators. All outcome assessments will be performed blinded and statistical analyses will be performed with the blinding intact.

\section{Safety}

Selenium is tolerated in short-term doses up to $10,000 \mu \mathrm{g}$ (that is, 100 experimental GRASS tablets). Acute intoxication is very rare and has only been related to accidental or suicidal intake $[4,21,22]$. Chronic intoxication requires long-term intake of at least $800 \mu \mathrm{g}$ per day [23], according to epidemiological studies in areas with very high selenium content in the soil. Thus, no signs of chronic intoxication have been observed in areas with daily intakes of up to about $800 \mu \mathrm{g}$ daily. In geographically highly exposed groups without signs of intoxication the serum concentrations have been reported to be 148 to $363 \mu \mathrm{g}$ per L [24] and 284 to $472 \mu$ g per L [23], respectively. In a study of $200 \mu \mathrm{g}$ selenium supplementation per day, the serum concentration reached a plateau at around $190 \mu \mathrm{g}$ per L [25].

According to the Danish National Food Institute, the 99th percentile for daily selenium intake through diet in Denmark is $93 \mu \mathrm{g}$ for men and $72 \mu \mathrm{g}$ for women. Therefore, a dosage of $200 \mu \mathrm{g}$ per day should not bring trial participants above the established upper tolerable intake of $300 \mu \mathrm{g}$ per day. A review of the safety of selenium supplementation with selenium yeast, which will be used in the GRASS trial [26], concludes that in about a dozen supplementation studies, none has shown evidence of toxicity even up to an intake of $800 \mu$ g selenium per day over a period of years. In conclusion, the experimental intervention with $200 \mu \mathrm{g}$ selenium per day is not expected to cause adverse reactions. Regardless, participants will be monitored for adverse events.

Overdosing can lead to gastrointestinal discomfort, hair and nail malformations and loss, peripheral neurological symptoms, fatigue and dizziness, and, in the case of very large selenium loads (1,000 times the daily trial dose), cardiovascular collapse and respiratory distress [21,22].

Assessment and reporting of adverse reactions (ARs): participants are prompted to self-report ARs at 6 and 12 weeks, and 6, 12 and 24 months, and are questioned about ARs at the study visits at 18 months of treatment and 12 months after stopping ATD treatment, respectively. ARs will be reported as a trial outcome. In addition, participants are instructed to contact their trial contact person if they experience symptoms suggestive of ARs.

Assessment and reporting of serious ARs (SARs), serious suspected SARs (SUSARs) and serious adverse events (SAEs): data on hospital admissions and mortality will be obtained through national registries at the end of the trial. Also, participants are informed and instructed to contact their trial contact person if they are admitted to a hospital for selenium intoxication, experience a clinical picture indicative of selenium intoxication, or experience a clinical picture that is unexpected but suspected to be related to selenium intoxication. When a possible serious event (SAE, SAR, or SUSAR) is identified, details will be sought from the patient's medical record and through direct contact with the patient. Any SAE, SAR or SUSAR will be reported as an outcome measure.

\section{Outcomes}

Outcomes will be assessed seven times during the trial (Table 1).

\section{Primary outcome}

The primary outcome is the proportion of participants with the composite outcome of ATD treatment failure in participants receiving ATD treatment during the last 12 months ( \pm 1 month) of the intervention period, who have had thyroid hyperfunction $(\mathrm{TSH}<0.1)$ during the last 12 months ( \pm 1 month) of the intervention period, or have been referred for ablative therapy (radioactive iodine or thyroid surgery) at some point during the entire intervention period.

\section{Secondary outcomes}

The secondary outcomes are each component of the primary outcome as follows: proportion of participants who receive ATD treatment (at any level) during the last 12 months ( \pm 1 month) of the intervention period (separate component of the primary outcome); proportion of participants who have thyroid hyperfunction $(\mathrm{TSH}<0.1)$ during the last 12 months ( \pm 1 month) of the intervention period (separate component of the primary outcome); proportion of participants who have been referred to ablative therapy (radioactive iodine or thyroid surgery) at some point during the entire intervention period (separate component of the primary outcome); thyroid-specific QoL during the first year after randomisation, and at the end of the intervention period ( 24 to 30 months), as measured by the global score in the ThyPRO questionnaire (Appendix 1); level of TRAb at 18 months, and at the end of the intervention period (24 to 30 months); hyperthyroid symptoms (ThyPRO subscale) during the first year after randomisation; eye symptoms (ThyPRO subscale) during the first year after randomisation, and at the end of the intervention period (24 to 30 months); number of patients with ARs during the intervention period, and number of patients with serious adverse events during the intervention period.

\section{Exploratory outcomes}

The following outcomes are of an exploratory nature: time to ATD treatment withdrawal (unsuccessful participants will be censored at 18 months); cost-effectiveness 
of the experimental intervention; incidence of Graves' orbitopathy during the intervention period, assessed as clinical activity score (CAS) $>1$ among patients with CAS scoring in the medical chart, and hypothyroid symptoms (ThyPRO subscale) during the intervention period.

\section{The trial data management system}

As a result of the pragmatic design with minimal participant-trial interaction, a large part of the data collection, trial conduct, and trial surveillance and timing is handled by the trial data management system. This trial data management system consists of a patientsurvey-interface, a trial-personnel-interface, a systemintegration interface and a programme motor. The system will be used for collection of outcomes, adverse events, and other trial-relevant information; for timing of trial events, that is, time for patient-reported outcomes and trial visits; for identification of need for actions (for example, contact to a participant); and for delivery of output to personnel or participants (for example, email notifications).

\section{Research biobank}

A research biobank for serum/plasma and urine samples will be established. The samples will initially be kept at each clinical trial site, and will later be analysed at central laboratories. Participants are informed verbally and in writing, and will consent to the withdrawal and storing of biological material in the GRASS trial. Any remaining samples will be transferred to a Biobank designated for future use. These samples will be used for genome-wide association studies (of predictors for remission and experimental intervention effects, or other indicators of autoimmunity), as may be specified in forthcoming protocols.

\section{Monitoring}

The trial will be monitored according to the International Committee of Harmonization ( $\mathrm{ICH}$ ) guidelines for good clinical research practice [27] by internal monitoring.

\section{Statistical analysis \\ Primary outcome: ATD treatment failure - sample size estimation}

Prior data indicate that the proportion of patients in the placebo group with ATD treatment failure is 50\% [28]. If the true proportion with ATD treatment failure is $37.5 \%$ among selenium-treated participants (that is, a relative risk reduction of $25 \%$ ), we will need to include 492 participants (246 experimental and 246 control participants) to be able to reject the null hypothesis with a power of $80 \%$ and a risk of type I error of $5 \%$.

The patient catchment areas of the participating centres include 1,640,000 persons. Assuming an annual incidence of Graves' hyperthyroidism of 40 per 100,000 after iodine fortification [1,29] and 50\% recurrence rate, the incident population (including recurrences) will be 788 patients annually. Assuming an inclusion of $45 \%$ of the potential patients, this will lead to about 29 participants per month and an inclusion period of about 17 months.

\section{Secondary outcomes - power estimation}

For the ThyPRO Global score, hyperthyroid symptoms and eye symptoms, if the true difference between experimental and the control participants is 5 (on a scale of 0 to 100 ) with SD of 20 , we will be able to reject the null hypothesis that the population means of the experimental and control groups are equal with a probability (power) of $79 \%$. The associated type I-risk is $5 \%$.

For the level of TRAb, if the true difference in TRAb between experimental and control participants is $0.15 \mathrm{IU}$ per L with SD of $0.5 \mathrm{IU}$ per $\mathrm{L}$, we will be able to reject the null hypothesis with a probability (power) of $91 \%$ and a type I error-risk of 5\%.

For the number of patients with adverse reactions, prior data indicate that the proportion of participants who experience adverse reactions in the control group is about 5\% [26]. If the true proportion of participants in the experimental group who experience adverse reactions is $10 \%$, we will be able to reject the null hypothesis that the failure rates for experimental and control participants are equal with probability (power) 50\%. The type I error probability associated with this test is $5 \%$.

\section{Data analysis}

All analyses will be intention-to-treat analyses with the intervention group concealed until two conclusions are drawn. The significance test will be at the $5 \%$ level and two-sided. Table 2 shows the priority for each outcome, when it will be measured, the mathematical type of measure, and the analytical procedure to be used when analyzing the outcome values.

\section{Analytical procedures}

Depending on the specific type of outcome measure, one of five types of regression analysis will be applied (Table 2). Indicator variable $\mathrm{I}$ ( 1 if $\mathrm{X}$ and 0 if $\mathrm{Y}$ ) is included as a covariate and the outcome measure (y) as the dependent variable. All analyses will be conducted both as unadjusted analyses and adjusted for stratification variables (clinical trial site and disease status (incident or relapse)). Discrepancies between the results of the two analyses will be discussed. For each covariate, an exploratory analysis, including the interaction between the covariate and the intervention indicator, will be conducted.

If the distribution of the primary outcome measure differs significantly between the two intervention groups, and the percentage of missing values is larger than $5 \%$, multiple imputations (MI) will be used. If so, the result obtained by imputation will be the primary result. In all 
Table 2 Outcome measures, their priorities, times of measurement, mathematical types and analytical categories (defining the statistical analysis to which they will be subjected)

\begin{tabular}{|c|c|c|c|}
\hline Outcome & Times of measurements & Type of quantity & Regression analysis to be applied \\
\hline \multicolumn{4}{|l|}{ Primary outcome } \\
\hline ATD treatment failure & End of trial & Binary & Logistic regression \\
\hline \multicolumn{4}{|l|}{ Secondary outcomes } \\
\hline $\begin{array}{l}\text { 1. ATD treatment within last } 12 \\
\text { months }\end{array}$ & End of trial & Binary & Logistic regression \\
\hline $\begin{array}{l}\text { 2. Thyroid hyperfunction after } \\
\text { ATD treatment withdrawal }\end{array}$ & End of trial & Binary & Logistic regression \\
\hline 3. Ablative therapy & End of trial & Binary & Logistic regression \\
\hline \multirow[t]{2}{*}{ 4. Global QoL ThyPRO score } & $\begin{array}{l}\text { a) Time sequence of five } \\
\text { measurements }\end{array}$ & Numerical & a) Mixed-model with repeated measures (MMRM) \\
\hline & $\begin{array}{l}\text { b) } 12 \text { months following ATD } \\
\text { treatment withdrawal }\end{array}$ & & $\begin{array}{l}\text { b) General linear univariate model. As sensitivity } \\
\text { analysis: Mann-Whitney test }\end{array}$ \\
\hline 5. Level of TRAb & $\begin{array}{l}\text { After } 18 \text { months and at the } \\
\text { end of intervention period }\end{array}$ & Numerical & $\begin{array}{l}\text { General linear univariate model. As sensitivity analysis: } \\
\text { Mann-Whitney test }\end{array}$ \\
\hline \multirow[t]{2}{*}{$\begin{array}{l}\text { 6. ThyPRO - hyperthyroid } \\
\text { symptoms }\end{array}$} & $\begin{array}{l}\text { a) Time sequence of five } \\
\text { measurements }^{\dagger}\end{array}$ & Numerical & a) Mixed-model with repeated measures (MMRM) \\
\hline & $\begin{array}{l}\text { b) } 12 \text { months following ATD } \\
\text { treatment withdrawal }{ }^{\ddagger}\end{array}$ & & $\begin{array}{l}\text { b) General linear univariate model. As sensitivity } \\
\text { analysis: Mann-Whitney test }\end{array}$ \\
\hline \multirow[t]{2}{*}{ 7. ThyPRO - eye symptoms } & $\begin{array}{l}\text { a) Time sequence of five } \\
\text { measurements }^{\dagger}\end{array}$ & Numerical & a) Mixed-model with repeated measures (MMRM) \\
\hline & $\begin{array}{l}\text { b) } 12 \text { months following ATD } \\
\text { treatment withdrawal }{ }^{\ddagger}\end{array}$ & & $\begin{array}{l}\text { b) General linear univariate model. As sensitivity } \\
\text { analysis: Mann-Whitney test }\end{array}$ \\
\hline 8. Adverse reactions & End of trial & $\begin{array}{l}\text { Rate }=\text { count/period of } \\
\text { intervention/day }\end{array}$ & $\begin{array}{l}\text { Generalised linear model, Poisson distribution, } \\
\text { link = log. As sensitivity analysis: Mann-Whitney test }\end{array}$ \\
\hline 9. Serious adverse reactions & End of trial & $\begin{array}{l}\text { Rate }=\text { count/period of } \\
\text { intervention/day }\end{array}$ & $\begin{array}{l}\text { Generalised linear model, Poisson distribution, } \\
\text { link = log. As sensitivity analysis: Mann-Whitney test }\end{array}$ \\
\hline \multicolumn{4}{|l|}{ Exploratory outcomes } \\
\hline 1. Time to ATD withdrawal ${ }^{\S}$ & End of trial & $\begin{array}{l}\text { Numerical (time until ATD } \\
\text { withdrawal (or censoring)) }\end{array}$ & $\begin{array}{l}\text { Cox proportional hazard rate model.As sensitivity } \\
\text { analysis: Kaplan-Meier estimates of survival function }\end{array}$ \\
\hline $\begin{array}{l}\text { 2. Incidence of Graves' } \\
\text { orbitopathy - CAS score }\end{array}$ & End of trial & Binary $($ CAS $>1)$ & Logistic regression \\
\hline \multirow[t]{2}{*}{$\begin{array}{l}\text { 3. ThyPRO - Hypothyroid } \\
\text { symptoms }\end{array}$} & $\begin{array}{l}\text { a) Time sequence of five } \\
\text { measurements }^{\dagger}\end{array}$ & Numerical & a) Mixed-model with repeated measures (MMRM) \\
\hline & $\begin{array}{l}\text { b) } 12 \text { months following ATD } \\
\text { treatment withdrawal }\end{array}$ & & $\begin{array}{l}\text { b) General linear univariate model. As sensitivity } \\
\text { analysis: MannWhitney test }\end{array}$ \\
\hline
\end{tabular}

${ }^{\dagger}$ This analysis (2) includes four measurements relative to the reference time (6 weeks, 12 weeks, 6 months and 12 months). ${ }^{*}$ This analysis ( 3 ) includes one measurement (12 months following ATD treatment withdrawal or failure to withdraw (alternative treatment instituted or deadline expired)) relative to a different reference time. ${ }^{5}$ End of ATD treatment or censoring at 18 months after randomisation or ablative surgery, provided the latter takes place during ATD treatment and prior to time of censoring. ATD, anti-thyroid drugs; QoL, quality of life; ThyPRO, thyroid patient-reported outcome; TRAb, TSH-receptor antibodies; CAS, clinical activity score.

events, the potential for bias caused by non-random missing values will be assessed using a worst- and best-case scenario. The gate keeping method of Dmitrienko et al. [30] will be used to adjust the observed $P$-values.

\section{Ethical considerations}

The GRASS trial has been approved by the Regional Ethics Committee (H-4-2012-026). The trial will be conducted in compliance with the guidelines of the latest Declaration of Helsinki and the International Conference on Harmonization (ICH) Good Clinical Practice (GCP) Guidelines [27].

\section{Publication plan}

The aim is to publish all results, positive, neutral, and negative, in peer-reviewed international journals. Authorship will be determined according to the guidelines from the International Committee of Medical Journal Editors [31].

\section{Discussion}

The design of the GRASS trial has faced three major challenges: the intent to conduct it as a pragmatic trial with as little interference with daily clinical management as possible, while still measuring QoL meticulously; the intent to comply with the $\mathrm{ICH}$ guidelines for GCP, even 
though the intervention is widely considered to be nontoxic and rather, a supplemental nutrient than a drug; and the need for a very long intervention period.

It was of great importance to the initiators of this trial, that the results would be directly applicable to daily clinical practice. Therefore, it was decided to conduct a pragmatic trial [32], with as little interference with daily clinical practice as possible. This could be accomplished by letting the patients follow their usual treatment at their usual hospitals by whichever physician was involved in their treatment. At the same time, we found it important to collect high quality data on the clinical course and QoL. We have, therefore, put great effort into the design of a trial management system, which could solve this schism of distance from participants compared to close monitoring. The system initiates and keeps track of patient input (QoL-measurements and other patient-reported outcomes), identifies need for trial personnel input and action, and collects data on thyroid function from medical chart systems. The system also identifies the site of the information-provider, as well as the disease status of new participants, and delivers randomisation codes stratified by site and disease status. Meticulous follow up on missing responses to the QoL measurements is incorporated into the system, to minimise the usual major problem with missing QoL data in clinical trials.

Somewhat similarly, the need to monitor adverse reactions and events in accordance with the ICH guidelines was in conflict with the intent to interfere as little as possible and with the fact that no previous selenium trial has identified any adverse reactions, which would also be quite surprising, given the wide therapeutic range of this dietary supplement. This is solved by a combination of thorough instruction of the participants to contact their trial person in case of symptoms indicative of adverse reactions or events, surveillance of patient-responses to prompts through the trial management system, and integration with national databases regarding hospitalizations.

The third issue was the decision to continue trial intervention until one year after stopping anti-thyroid drugs (ATD treatment withdrawal). Patients are not considered in remission unless they are still euthyroid one year after stopping medication. Since selenium is considered to have an attenuating effect on thyroid autoimmunity, a long duration of treatment was considered necessary to test our hypothesis, that is, that selenium can indeed lead to more patients staying in remission (and not just reaching euthyroidism faster and obtaining better QoL).

\section{Trial status}

The first patient was enrolled in December 2012.

\section{Abbreviations}

ATD: Anti-thyroid drug; AR: Adverse reaction; CAS: Clinical activity score:

CRF: Case report form; GCP: Good Clinical Practice; ICH: International
Conference on Harmonization; QoL: Quality of life; SAE: Serious adverse event; SAR: Serious adverse reaction; s-Se: Serum selenium; SmPC: Summary of products characteristics; SUSAR: Suspected serious adverse reaction; $\mathrm{T}_{3}$ : Triiodothyronine; $\mathrm{T}_{4}$ : Thyroxine; ThyPRO: Thyroid patient related outcome (thyroid-specific quality of life questionnaire); TPOAb: Thyroid peroxidase antibody; TSH: Thyroid stimulating hormone; TRAb: Thyroid stimulating hormone receptor antibody (TSH receptor antibody).

\section{Competing interest}

None of the investigators have any financial or non-financial competing interest.

\section{Author contributions}

All authors contributed to the design of the trial, preparation and review of the manuscript and all authors read and approved the final manuscript.

\section{Funding}

The GRASS trial is part of the ThyQoL project, a strategic project headed by Ulla Feldt-Rasmussen and funded by the The Danish Agency for Science, Technology and Innovation (grant 271-09-0143). It is also part of a grant given to Torquil Watt by the Danish Council for Independent Research (grant 09-066886).

\section{Author details}

'Department of Medical Endocrinology, Copenhagen University Hospital Rigshospitalet, Blegdamsvej 9, DK-2100 Copenhagen, Denmark. ²National Research Centre for the Working Environment, Copenhagen, Denmark.

${ }^{3}$ Institute of Public Health Science, University of Copenhagen, Copenhagen, Denmark. ${ }^{4}$ Department of Endocrinology and Metabolism, Odense University Hospital, Odense, Denmark. ${ }^{5}$ Copenhagen Trial Unit, Copenhagen University Hospital Rigshospitalet, Copenhagen, Denmark. ${ }^{6}$ Department of Endocrinology, Hospital of Southwest Denmark Esbjerg, Esbjerg, Denmark. 7Department of Endocrinology and Gastroenterology, Copenhagen University Hospital Bispebjerg, Copenhagen, Denmark. ${ }^{8}$ Department of Endocrinology, Copenhagen University Hospital Hvidovre, Copenhagen, Denmark. ${ }^{9}$ Department of Cardiology and Endocrinology, Endocrine Unit, Copenhagen University Hospital Hillerød, Hillerød, Denmark. ${ }^{10}$ Department of Internal Medicine, Endocrine Unit, Copenhagen University Hospital Herlev, Copenhagen, Denmark. ${ }^{11}$ Glostrup Research Institute, Copenhagen University Hospital Glostrup, Copenhagen, Denmark.

Received: 4 September 2012 Accepted: 10 April 2013

Published: 30 April 2013

\section{References}

1. Cerqueira C, Knudsen N, Ovesen L, Perrild H, Rasmussen LB, Laurberg P, Jorgensen T: Association of iodine fortification with incident use of antithyroid medication-a Danish Nationwide Study. I Clin Endocrinol Metab 2009, 94:2400-2405

2. Hegedus L: Treatment of Graves' hyperthyroidism: evidence-based and emerging modalities. Endocrinol Metab Clin North Am 2009, 38:355-71.

3. Watt T, Groenvold M, Rasmussen AK, Bonnema SJ, Hegedüs L, Bjorner JB, Feldt-Rasmussen U: Quality of life in patients with benign thyroid disorders. A review. Eur J Endocrinol 2006, 154:501-510.

4. Rasmussen LB, Mejborn H, Andersen NL, Dragsted LO, Krogholm KS, Larsen EH, et al: Selen og Sundhed. Copenhagen: Danmarks Fødevareforskning; 2006.

5. Duntas LH: Selenium and the thyroid: a close-knit connection. J Clin Endocrinol Metab 2010, 95:5180-5188.

6. Food and Nutrition Board loM: Dietary reference intakes for vitamin $C$, vitamin E, selenium and carotenoids. In Food and Nutrition Board, I.O.M. Edited by Poillon F, Newberry S. Washington, D.C: National Academy Press; 2000:1.

7. Scientific Committee on Food: Opinion of the Scientific Committee on Food on the Tolerable Upper Intake Level for Selenium. 1. 2000. Brussels: European Commission. Scientific Committee on Food; 2000.

8. Marcocci C, Leo M, Altea MA: Oxidative stress in Graves' disease. European Thyroid Journal 2012, 1:80-87.

9. Duntas LH: The role of selenium in thyroid autoimmunity and cancer. Thyroid 2006, 16:455-460.

10. Marcocci C, Kahaly GJ, Krassas GE, Bartalena L, Prummel M, Stahl M, Altea MA, Nardi M, Pitz S, Boboridis K, Sivelli P, von AG, Mourits MP, 
Baldeschi L, Bencivelli W, Wiersinga W: Selenium and the course of mild Graves' orbitopathy. N Engl J Med 2011, 364:1920-1931.

11. Vrca VB, Skreb F, Cepelak I, Romic Z, Mayer L: Supplementation with antioxidants in the treatment of Graves' disease; the effect on glutathione peroxidase activity and concentration of selenium. Clin Chim Acta 2004, 341:55-63.

12. Vrca VB, Skreb F, Cepelak I, Mayer L: Supplementation with antioxidants in the treatment of Graves' disease: the effect on the extracellular antioxidative parameters. Acta Pharm 2004, 54:79-89.

13. Wertenbruch T, Willenberg HS, Sagert C, Nguyen TB, Bahlo M, Feldkamp J, Groeger C, Hermsen D, Scherbaum WA, Schott M: Serum selenium levels in patients with remission and relapse of graves' disease. Med Chem 2007, 3:281-284.

14. Gartner R, Gasnier BC, Dietrich JW, Krebs B, Angstwurm MW: Selenium supplementation in patients with autoimmune thyroiditis decreases thyroid peroxidase antibodies concentrations. J Clin Endocrinol Metab 2002, 87:1687-1691.

15. Gartner R, Gasnier BC: Selenium in the treatment of autoimmune thyroiditis. Biofactors 2003, 19:165-170.

16. Duntas $L H$, Mantzou E, Koutras DA: Effects of a six month treatment with selenomethionine in patients with autoimmune thyroiditis. Eur J Endocrinol 2003, 148:389-393.

17. Turker O, Kumanlioglu K, Karapolat I, Dogan I: Selenium treatment in autoimmune thyroiditis: 9-month follow-up with variable doses. J Endocrinol 2006, 190:151-156.

18. Mazokopakis EE, Papadakis JA, Papadomanolaki MG, Batistakis AG, Giannakopoulos TG, Protopapadakis EE, Ganotakis ES: Effects of 12 months treatment with L-selenomethionine on serum anti-TPO Levels in Patients with Hashimoto's thyroiditis. Thyroid 2007, 17:609-612.

19. Nacamulli D, Mian C, Petricca D, Lazzarotto F, Barollo S, Pozza D, Masiero S, Faggian D, Plebani M, Girelli ME, Mantero F, Betterle C: Influence of physiological dietary selenium supplementation on the natural course of autoimmune thyroiditis. Clin Endocrinol (Oxf) 2010, 73:535-539.

20. Karanikas G, Schuetz M, Kontur S, Duan H, Kommata S, Schoen R, Antoni A Kletter K, Dudczak R, Willheim M: No immunological benefit of selenium in consecutive patients with autoimmune thyroiditis. Thyroid 2008, 18:7-12

21. Pentel $P$, Fletcher $D$, Jentzen J: Fatal acute selenium toxicity. J Forensic SCi 1985, 30:556-562

22. Högberg J, Alexander J: Selenium. In Handbook on the toxicology of metals. Edited by Friberg L, Nordberg GF, Vouk VB. Amsterdam: Elsevier; 1986:482-520.

23. Yang $G Q$, Wang $S Z$, Zhou RH, Sun SZ: Endemic selenium intoxication of humans in China. Am J Clin Nutr 1983, 37:872-881.

24. Longnecker MP, Taylor PR, Levander OA, Howe M, Veillon C, McAdam PA, Patterson KY, Holden JM, Stampfer MJ, Morris JS: Selenium in diet, blood, and toenails in relation to human health in a seleniferous area. Am J Clin Nutr 1991, 53:1288-1294.

25. Clark LC, Combs GF Jr, Turnbull BW, Slate EH, Chalker DK, Chow J, Davis LS Glover RA, Graham GF, Gross EG, Krongrad A, Lesher JL Jr, Park HK, Sanders BB Jr, Smith CL, Taylor JR: Effects of selenium supplementation for cancer prevention in patients with carcinoma of the skin. A randomized controlled trial. Nutritional Prevention of Cancer Study Group. JAMA 1996, 276:1957-1963.

26. Rayman MP: The use of high-selenium yeast to raise selenium status: how does it measure up? Br J Nutr 2004, 92:557-573.

27. International Conference on Harmonisation Guideline for International conference on harmonisation of technical requirements for registration of pharmaceuticals for human use: Guideline for Good Clinical Practice E6 (R1). ICH Harmonised Tripartite Guideline. 10-6-1996. http://www.ich.org/fileadmin/ Public_Web_Site/ICH_Products/Guidelines/Efficacy/E6_R1/Step4/ E6_R1_Guideline.pdf.

28. Dupont WD, Plummer WD Jr: Power and sample size calculations. A review and computer program. Control Clin Trials 1990, 11:116-128.

29. Lantz M, Abraham-Nordling M, Svensson J, Allin G, Allengren B: Immigration and the incidence of Graves' thyrotoxicosis, thyrotoxic multinodular goiter and solitary toxic adenoma. Eur J Endocrinol 2009, 160:201-206.

30. Dmitrienko A, Tamhane AC, Wiens BL: General multistage gatekeeping procedures. Biom J 2008, 50:667-677.
31. International Committee of Medical Journal Editors: Uniform Requirements for Manuscripts Submitted to Biomedical Journals: Ethical Considerations in the Conduct and Reporting of Research: Authorship and Contributorship. 2010. http://www.icmje.org/ethical_1author.html.

32. Ware JH, Hamel MB: Pragmatic trials-guides to better patient care? N Engl J Med 2011, 364:1685-1687.

doi:10.1186/1745-6215-14-119

Cite this article as: Watt et al: Selenium supplementation for patients with Graves' hyperthyroidism (the GRASS trial): study protocol for a randomized controlled trial. Trials 2013 14:119.

\section{Submit your next manuscript to BioMed Central and take full advantage of:}

- Convenient online submission

- Thorough peer review

- No space constraints or color figure charges

- Immediate publication on acceptance

- Inclusion in PubMed, CAS, Scopus and Google Scholar

- Research which is freely available for redistribution 